This document is published in:

Demazeau, Y.; et al. (eds.) (2010) Trends in Practical Applications of Agents and Multiagent Systems: 8th International Conference on Practical Applications of Agents and Multiagent Systems, (Advances in Intelligent and Soft Computing, 71) Springer, 493-500.

DOI: http://dx.doi.org/10.1007/978-3-642-12433-4_58

(C) 2010 Springer-Verlag Berlin Heidelberg 


\title{
Multi-agent System (MAS) Applications in Ambient Intelligence (AmI) Environments
}

\author{
Nayat Sánchez-Pi, Eleni Mangina, Javier Carbó, and José Manuel Molina
}

\begin{abstract}
Research in context-aware systems has been moving towards reusable and adaptable architectures for managing more advanced human-computer interfaces. Ambient. Intelligence (AmI) investigates computer-based services, which are ubiquitous and based on a variety of objects and devices. Their intelligent and intuitive interfaces act as mediators through which people can interact with the ambient environment. In this paper we present an agent-based architecture which supports the execution of agents in AmI environments. Two case studies are also presented, an airport information system and a railway information system, which uses spoken conversational agents to respond to the user's requests using the contextual information that includes the location information of the user.
\end{abstract}

Keywords: Multi-agent systems, Services Oriented Architectures, Mobile Context-Aware Systems, Multi-Agent Systems.

\section{Introduction}

Mobile technology is increasingly entering in all aspects of our life and in all sectors, opening a world of unprecedented scenarios where people interact with electronic devices embedded in environments that are sensitive to the presence of users. These context-aware environments combine ubiquitous information, communication, with enhanced personalization, natural interaction and intelligence.

Nayat Sánchez-Pi · Javier Carbó · José Manuel Molina

Computer Science Department

Carlos III University of Madrid

Avda de la Universidad Carlos III, 22. 28270. Colmenarejo, Spain

e-mail: \{nayat. sanchez, javier. carbo, josemanuel.molina\} @uc $3 \mathrm{~m}$. es

Eleni Mangina

Computer Science Department

University College of Dublin

Dublin, Ireland

e-mail: \{eleni.mangina\} @ucd.ie 
The use of this context offers the possibility to tailor a new type of advanced applications. The design and development of effective applications should definitely take into account the characteristics of the context from which a service is requested [9]. Contextual information can be either the type of the device exploited to access a service, or the location of the user, or its personal preferences, etc.

Although there is not a complete agreement on the definition of context, the most widely accepted is the one proposed by [3]: "Any information that can be used to characterize the situation of an entity is relevant to the interaction between a user and an application, including the user and the application themselves". As can be observed from this definition, any information source can be considered context as long as it provides knowledge relevant to handle the communication between the user and the system. In addition, the user is also considered to be part of the contextual information. Kang et al [15] differentiate two types of context: internal and external. The former describes the user state (e.g. communication context and emotional state), whereas the latter refers to the environment state (e.g. location and temporal context). Most studies in the literature focus on the external context. However, although external information, such as location, can be a good indicator of the user intentions in some domains, in many applications it is necessary to take into account more complex information sources about the user state, such as emotional status [1] or social information [17]. External and internal context are intimately related, as can be seen in representative examples like service context and proactive systems.

Context information can be gathered from a wide variety of sources, which produces heterogeneity in terms of quality and persistence. As described in [11], static context deals with invariant features, whereas dynamic context is able to cope with information that changes. The frequency of such changes is very variable and can deeply influence the way in which context is obtained. It is reasonable to obtain largely static context directly from users, and frequently changing context from indirect means such as sensors.

Ambient Intelligence is associated to a society based on unobtrusive, often invisible interactions amongst people and computer-based services taking place in a global computing environment. A good point seen on the AmI vision is that the electronic or digital part of the ambience (devices) will often need to act intelligently on behalf of people [21]. In [22], O'Hare et al. advocate the use of agents as a key enabler in the delivery of ambient intelligence and ubiquitous environments. The components of ambience will need to be both reactive and proactive, behaving as if they were agents that act on behalf of people. If we assume that agents are abstractions for the interaction within an ambient intelligent environment, one aspect that we need to ensure is that their behavior is regulated and coordinated. For this purpose, we need rules that take into consideration the context (location, user profile, type of device, etc...) in which these interactions take place. Taking care this, the system needs an organization similar to the one envisaged by artificial agent societies. The society is there not only to regulate behavior but also to distribute responsibility amongst the member agents.

This paper is structured as follows: Section 2 describes the main characteristics of our agent-based architecture for providing context-aware adaptable systems and 
the new characteristics implemented; Section 3 presents the case of use in the domain of an airport; Section 4 presents another case of use with an intelligent speech-based interface in a railway domain and finally Section 5 present our conclusions and future work.

\section{MAS Architecture for AmI}

Current research has focused on agent and multi-agent implementations which support the ubiquitous provisioning of context-aware services to mobile devices. CONSORT [16] is a multi-agent architecture including a middle agent that translate sensor based raw representation of the locations into a conceptual one using a location ontology in two application domains that model the geographical space in a cognitive way: an intelligent assistant at a museum and a wireless-LAN based location system. Another is SMAUG [19] which is a multi-agent context-aware system that allows tutors and pupils of a university to fully manage their activities. SMAUG offers its users with context-aware information from their environment and also gives them a location service to physically locate every user of the system. Another one is IUMELA [18], is an intelligent modular-education learning assistant designed using a multi-agent systems in order to assist students in their career decision-making process. All the above research projects although successful to their own problem domain, do not provide a general architecture that can be applied to different scenarios and case studies. Within this paper our aim is to present the redesign our initial multi-agent system for AmI [20; 21], utilized in two different case studies. The redesign includes new features to support an intelligent speech-based interface.

Figure 1 shows the architecture of our previous work, while Figure 2 denotes our new proposal that allow us distribute contextual information between different agents.

New agent's functionalities state as follow:

Positioning agent main tasks rely on the user identification and user location into the environment. In order to do this, this agent needs to connect to Aruba Positioning System [20], to read the positioning information. This information consists of an (x,y) coordinates and the building and floor information of the system. Facilitator agent is the responsible of the services management and the discovering of services agent identification. Therefore, it carries out the matching between the user profile stored in the user agent and the different services, since they can reach an agreement with clients and communicate the most suitable services to them according clients preferences and profile. User agents main goals includes negotiation with the facilitator agent, recommend services to other user agents, trust in other agents, and manage and improve their internal profile to receive better services according to it. The information defined in the user profile stored in the user agent can be classified into two different groups:

-Personal information: user's name, gender, age, current language, skill level when interacting with dialog systems, pathologies or speech disorders;

-User preferences: This set is split into different statistic groups, which are defined according to a specific domain-knowledge. This new subdivision allows the 


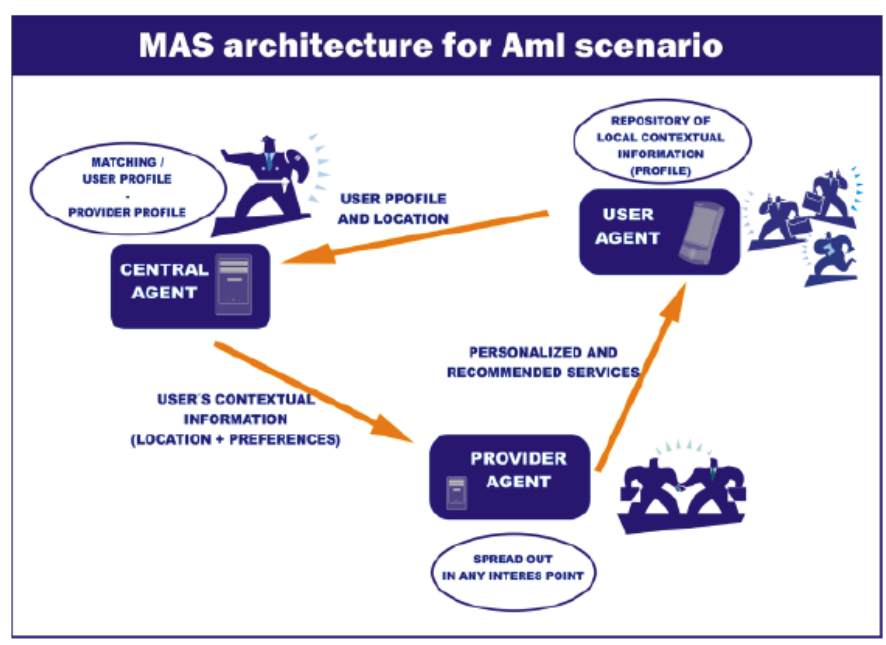

Fig. 1 MAS architecture (extracted from V. Fuentes, N. Sanchez-Pi, J. Carbó, J.M. Molina. Designing a Distributed Context-Aware Multi-Agent System" from Agent-Based Ubiquitous Computing, chapter of Agent-based Ubiquitous Computing, Series: Atlantis Ambient and Pervasive Intelligence - Vol. 1. E. Mangina, J. Carbó, J.M.Molina (Eds.). Ed. World Scientific. ISBN 978-90-78677-10-9).

system to infer the preferences of each user regarding specific queries included in the task (i.e. in a railway information system to know that a specific user usually requires timetables information) or relative to specific values of attributes to be used in these queries (i.e. the preferences for travelling during a specific part of the day or by using a specific type of train).

Although there are a high variety of applications in which conversational agents can be used. One of the most wide-spread is information retrieval that was the main reason to include conversational agents in this proposal.

To successfully manage the interaction with the users, conversational agents usually carry out five main tasks: automatic speech recognition (ASR), natural language understanding (NLU), dialog management (DM), natural language generation (NLG) and text-to-speech synthesis (TTS). These tasks are usually implemented in different modules.

According to the implementation and although other alternatives exist, JADELEAP agent platform was chosen since it is a Java-based and FIPA compliant agent platform, where agents communicate by sending FIPA ACL messages over a TCP/IP connection between different runtime environments on local servers or running on wireless devices. One local server hosts the positioning agent that rules over all the sensors of the domain (these sensors are just simulated inside the implementation of the central agent) that provide location information of users. Another server hosts the facilitator agent that matches the user profile with the services while one or more local servers host conversational agents acting on behalf of the different services. Each portable client device runs a JADE-LEAP 


\section{MAS supporting Speech-based Interface}

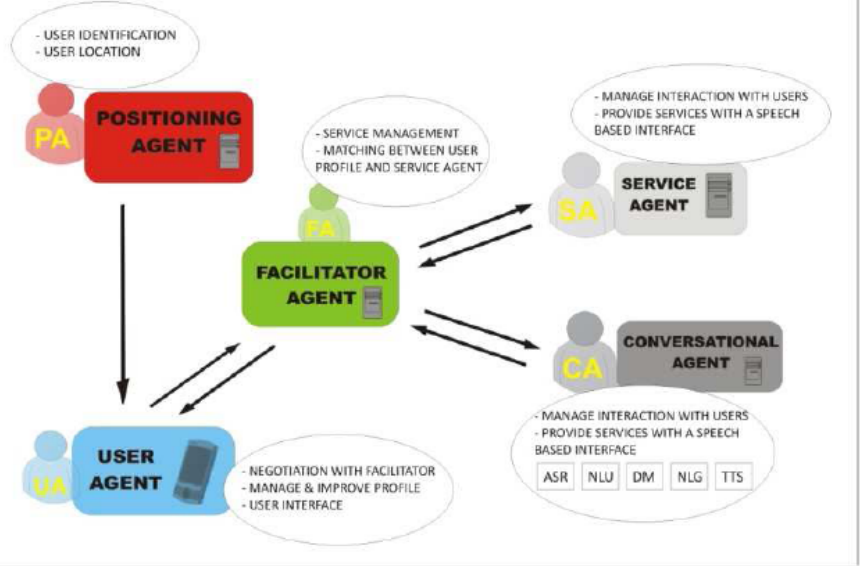

Fig. 2 MAS adequacy proposed new architecture to support speech-based interface

container that hosts one single agent that is used to provide a way to interact with the user (through a GUI), so the user can be informed and interact with the other agents running on different servers. JADE/LEAP platform logically connects the agents on the different servers with each other and with the corresponding user agents running on the users' devices.

\section{Case Study I: Airport Scenario}

The first case study of the proposed new architecture, presents the definition of an information system for an airport domain. What we precisely propose is a distributed approach based on agents to address the problem of using context-aware information to offer customized services to users in an airport domain, and especially for the Barajas-Madrid Airport and for services offered to clients, passengers, crew and staff of IBERIA airline. We are familiar to this problem since we previously developed a centralized system with the same final intention using Appear Networks Platform and Aruba Wi-Fi Location System [20; 21]. The current proposal includes agents implemented in JADE and LEAP that will make use of the user profiles to customize and recommend different services to other agents avoiding an obtrusive participation of a central server. First we assume an initial minimal profile known user agent: name, agent role, passport number, nationality and travel info (flight numbers, companies, origin and target). Figure 3 shows the console agents and the HTC touch terminal. We will describe how the system works by following all the messages exchanges when a passenger "Paul" arrives to the airport.When Paul enters into the system's wireless network. Aruba Positioning system discovers the Paul's agent position. Later, positioning agent provides Aruba positioning information to the Paul's user agent. Once Paul's user 
agent (running the LEAP code on the mobile device) knows its location sends it to the facilitator agent as well as the information regarding using a specific kind of service, in this case Paul's preferences include automated check-in service. After that, facilitator agent sends the automated check-in agent identification, the interaction between them begin and the provisioning of the service occur.

\section{Case Study II: Railway Station Scenario}

There is a high variety of applications in which conversational agents can be used. One of the most wide-spread is information retrieval. Some sample applications are tourist and travel information [8, 14], weather forecast over the phone [23], speech controlled telephone banking systems $[12,13]$, conference help $[5,10]$, etc. They have also been used for education and training, particularly in improving phonetic and linguistic skills: assistance and guidance to F18 aircraft personnel during maintenance tasks [4], dialog applications for computer-aided speech therapy with different language pathologies [2].

In this section we present a railway information system that includes a speechbased interface. We are familiar to this problem since we previously developed a centralized system with the same final intention using Appear Networks Platform and Aruba Wi-Fi Location System [6, 7]. To successfully manage the interaction with the users, conversational agents usually carry out five main tasks: automatic speech recognition (ASR), natural language understanding (NLU), dialog management (DM), natural language generation (NLG) and text-to-speech synthesis (TTS). These tasks are usually implemented in different modules.

The behaviour of the system is the following: In the first phase, the Aruba Positioning system discovers the user's position while he enters the Wi-Fi network in the Atocha Station. Later, positioning agent provides Aruba positioning information to the user agent. Once user agent knows its location sends it to the facilitator agent as well as the information regarding using a specific kind of service, in this case the user decides to ask for a conversational service. Following the set of phases, a conversational agent that provides the railway information and has been previously detected, asks the user agent about context information to be used during the interaction to provide the personalized service. Once this context information is received by the Context Manager included in the conversational agent, it loads the specific context profile characteristics. This information is then consulted by the rest of the modules in the conversational agent to personalize the provided service.

\section{Conclusions}

In this paper we presented middleware architecture for AmI environment to manage location-sensing systems and dynamically deploying services at suitable computing devices. Although it is a general framework in the sense that it is independent of any higher-level applications and location-sensing systems, we have designed and implemented a prototype system of the infrastructure and demonstrated its effectiveness in two practical applications: an airport and railway 
scenario. The main contributions are related to the adaptation of the MAS architecture. We have also presented two case studies: an airport information system and a railway information system, which uses spoken conversational agents to respond to the user's requests using the contextual information that includes the location information of the user.

Finally, we would like to point out further issues to be resolved. Since the framework presented in this paper has been applied to specific applications, we plan to evaluate it in terms of utility function of the MAS. The utility function will give a quantitative result of the effectiveness of the updated architecture compared to the initially implemented one. As a future work, we would like to evaluate our methodology in real environments.

Acknowledgements. This work has been partially supported by CICYT TIN2008-06742C02-02/TSI, CICYT TEC2008-06732-C02-02/TEC, SINPROB, CAM MADRINET S-0505/TIC/0255 and DPS2008-07029-C02-02.

\section{References}

1. Callejas, Z., López-Cózar, R.: Influence of contextual information in emotion annotation for spoken dialogue systems. Speech Communication 50(5), 416-433 (2008)

2. Vaquero, C., Saz, O., Lleida, E., Marcos, J., Canalís, C.: VOCALIZA: An application for computer-aided speech therapy in Spanish language. In: Proc. of IV Jornadas en Tecnología del Habla, Zaragoza, Spain, pp. 321-326 (2006)

3. Dey, A., Abowd, G.: Towards a better understanding of context and contextawareness. In: Proc. of the 2000 Conference on Human Factors in Computer Systems (CHI 2000), New York, New Jersey, US, pp. 304-307 (2000)

4. Bohus, D., Rudnicky, A.: LARRI: A Language-Based Maintenance and Repair Assistant. In: Proc. of Multi-Modal Dialogue in Mobile Environments Conference (IDS 2002), Kloster Irsee, Germany, pp. 203-218 (2002)

5. Bohus, D., Grau, S., Huggins-Daines, D., Keri, V., Krishna, G., Kumar, R., Raux, A., Tomko, S.: Conquest - an Open-Source Dialog System for Conferences. In: Proc. of Human Language Technologies 2007: The Conference of the North American Chapter of the Association for Computational Linguistics, Rochester, NY, USA, pp. 9-12 (2007)

6. Griol, D., Sánchez-Pi, N., Carbó, J., Molina, J.M.: A Context-Aware Architecture to Provide Adaptive Services by means of Spoken Dialogue Interaction. In: Proc of ICAI 2009 - The 2009 International Conference on Artificial Intelligence, Las Vegas, USA (July 2009)

7. Griol, D., Sánchez-Pi, N., Carbó, J., Molina, J.M.: Context-Aware Approach for Orally Accessible Web Services. In: Proc. of IEEE/WIC/ACM International Joint Conferences on Web Intelligence and Intelligent Agent Technologies, Milán, Italy, September 2009, pp. 171-174 (2009)

8. den Os, E., Boves, L., Lamel, L., Baggia, P.: Overview of the ARISE project. In: Proc. of the European Conference on Speech Technology (Eurospeech 1999), Budapest, Hungary, pp. 1527-1530 (1999) 
9. Fuentes, V., Pi, N.S., Carbó, J., Molina, J.M.: Reputation in user profiling for a context-aware multiagent system. In: Proc. of the 4th European Workshop on Multi-Agent Systems, EUMAS 2006, Lisbon, Portugal (2006)

10. Andeani, G., Fabbrizio, D.D., Gilbert, M., Gillick, D., Hakkani-Tur, D., Lemon, O.: Let's DISCOH: Collecting an Annotated Open Corpus with Dialogue Acts and Reward Signals for Natural Language Helpdesks. In: Proc. of IEEE 2006 Workshop on Spoken Language Technology (SLT 2006), Palm Beach, Aruba, pp. 218-221 (2006)

11. Henricksen, K., Indulska, J., Rakotonirainy, A.: Modeling context information in pervasive computing systems. In: Mattern, F., Naghshineh, M. (eds.) PERVASIVE 2002. LNCS, vol. 2414, pp. 167-180. Springer, Heidelberg (2002)

12. Hardy, H., Biermann, A., Inouye, R., McKenzie, A., Strzalkowski, T., Ursu, C., Webb, N., Wu, M.: The Amitiés system: Data-driven techniques for automated dialogue. Speech Communication 48, 354-373 (2006)

13. Melin, H., Sandell, A., Ihse, M.: Ctt-bank: A speech controlled telephone banking system - an initial evaluation. In: TMH-QPSR, vol. 1, pp. 1-27 (2001)

14. Glass, J., Flammia, G., Goodine, D., Phillips, M., Polifroni, J., Sakai, S., Sene ${ }^{\circledR}$, S., Zue, V.: Multilingual spoken-language understanding in the MIT Voyager system. Speech Communication 17, 1-18 (1995)

15. Kang, H., Suh, E., Yoo, K.: Packet-based context aware system to determine information system user's context. Expert Systems with Applications 35, 286-300 (2008)

16. Kurumatani, K., Sashima, A., Izumi, N.: Location-mediated web services coordination in ubiquitous computing. In: IEEE Int. Conf. on Web Services (ICWS 2004), pp. 822823 (2004)

17. Markopoulos, P., de Ruyter, B., Privender, S., van Breemen, A.: Case study: bringing social intelligence into home dialogue systems. Interactions 12(4), 37-44 (2005)

18. McGovern, E., Roche, B., Collier, R., Mangina, E.: IUMELA: A Lightweight MultiAgent Systems Based Mobile Learning Assistant Using the ABITS Messaging Service. In: Indulska, J., Ma, J., Yang, L.T., Ungerer, T., Cao, J. (eds.) UIC 2007. LNCS, vol. 4611, pp. 1056-1065. Springer, Heidelberg (2007)

19. Nieto-Carvajal, I., Botía, J.A., Ruiz, P.M., Gómez-Skarmeta, A.F.: Implementation and Evaluation of a Location-Aware Wireless Multi-agent System. In: Yang, L.T., Guo, M., Gao, G.R., Jha, N.K. (eds.) EUC 2004. LNCS, vol. 3207, pp. 528-537. Springer, Heidelberg (2004)

20. Sánchez-Pi, N., Carbó, J., Molina, J.: Analysis and Design of a Multi-Agent System Using Gaia Methodology in an Airport Case of Use. In: Proc. of IBERAGENTS 2008. 7th Ibero-American Workshop in Multi-Agent Systems. In Conjunction with IBERAMIA 2008, Lisbon, Portugal (October 2008)

21. Sánchez-Pi, N., Carbó, J., Molina, J.: JADE/LEAP agents in an aml domain. In: Corchado, E., Abraham, A., Pedrycz, W. (eds.) HAIS 2008. LNCS (LNAI), vol. 5271, pp. 62-69. Springer, Heidelberg (2008)

22. O'Hare, G.M.P., O’Grady, M.J., Kegan, S., O’Kane, D., Tynan, R., Marsh, D.: Inteligent Agile Agents: Active Enablers for Ambient Intelligence. In: ACM's Special Interest Group on Computer-Human Interaction (SIGCHI), Ambient Intelligence for Scientific Discovery (AISD) Workshop, Vienna, April 25 (2004)

23. Zue, V., Sener, S., Glass, J., Polifroni, J., Pao, C., Hazen, T., Hetherington, L.: JUPITER: A telephone-based conversational interface for weather information. IEEE Transactions on Speech and Audio Processing 8, 85-96 (2000) 